\title{
Análisis del Grado de Formación en Primeros Auxilios del Profesorado en Activo de Educación Infantil y Primaria
}

\author{
Zuriñe Gaintza(1) y Zaloa Velasco(2) \\ (1) Universidad del País Vasco / Euskal Herriko Unibertsitatea, Escuela de magisterio, Departamento de \\ Didáctica y Organización Escolar, Barrio Sarriena s/n 48940 Leioa-España (e-mail: zuri.gaintza@ehu.eus) \\ (2) Servicio Vasco de Salud / Osakidetza, Larretxe 10, 48650 Barrika-España \\ (e-mail: zaloa.velascocarneros@osakidetza.eus)
}

Recibido Ago. 2, 2016; Aceptado Oct. 5, 2016; Versión final Nov. 12, 2016, Publicado Abr. 2017

\begin{abstract}
Resumen
El objetivo de este artículo es analizar el conocimiento que sobre primeros auxilios posee el profesorado de educación infantil y primaria. Numerosos accidentes ocurren cada año en los centros escolares, por lo que conocer la capacidad del profesorado para responder ante situaciones de urgencia resulta esencial. Se aplica una prueba estandarizada a un total de 361 docentes. Los resultados muestran que, en general, el profesorado no está adecuadamente cualificado para atender situaciones de urgencia en la escuela, aunque se constatan diferencias significativas entre quienes han recibido formación sobre el tema y los que no. Tras llevar a cabo análisis jerárquicos se obtienen diferentes perfiles del profesorado con distintos grados de formación. Las conclusiones sugieren la necesidad de incorporar formación sobre primeros auxilios en los estudios de grado de magisterio, así como de ofertar cursos de formación permanente que pongan especial atención en la reanimación cardiopulmonar.
\end{abstract}

Palabras clave: primeros auxilios; formación del profesorado; educación infantil; educación primaria; grado de magisterio

\section{Analysis of the Training Level on First Aids of Currently Employed Teachers of Pre-School and Primary School}

\begin{abstract}
The objective of this paper is, specifically, to analyze the knowledge that teachers of pre-school and primary school have on first aids. Many accidents occur each year in schools, so it is essential to know teachers' ability to act properly in case of emergency. A standardized test was conducted on a sample of 361 teachers. The results show that, in general, teachers are not enough qualified to respond correctly in the event of emergency in the school. However, there are some significant differences between those that have been trained on this subject and those that have not. After conducting a hierarchical analysis, some profiles of teachers with different training level were shown. Conclusions suggest that it is necessary to incorporate first aids training in teacher education degree, as well as to offer continuous education courses, especially focused on cardiopulmonary resuscitation.
\end{abstract}

Keywords: first aids; teacher training; pre-school education; primary education; teacher education degree 


\section{INTRODUCCIÓN}

Los niños y niñas entre edades comprendidas entre los 3 y los 12 años, pasan gran parte de su vida en la escuela. Esta realidad, nos lleva a pensar que en este rango de edad la probabilidad de que ocurra un accidente en el ámbito escolar es bastante alta. De hecho, en los países desarrollados los accidentes escolares se encuentran entre las primeras causas de defunción infantil (World Health Organization, 2006). Estudios realizados en varias comunidades autónomas de España encuentran que los accidentes escolares son una realidad en las escuelas. Así, en Cataluña, la escuela aparece como el segundo espacio con mayor incidencia de sucesos (Brugulat, Medina y Mompart, 2009). En la Comunidad Autónoma del País Vasco, ámbito de este estudio, de las 17.686 lesiones no intencionadas acaecidas en menores de 14 años, el $14.5 \%$ se produjo en el centro de estudios (Osakidetza, 2013). La encuesta de Salud de la Comunidad Valenciana (2010) recoge que la mayor parte de los accidentes para la población menor de 16 años, tiene lugar en el entorno escolar (31\%). En Cantabria (Gautier y Martínez, 2009) y en Cataluña (Longás E., Longás, J. y Riera i Romaní, 2014) concluyen que en torno a un 5\% de los niños y niñas sufren accidentes y lesiones en el contexto escolar.

Aunque la palabra accidente parece hacer alusión a algo imprevisto, inevitable y fortuito, cabe incidir en que "la adopción de ciertos hábitos sencillos, puede contribuir a reducirlos" (Mapfre, 2014, p. 7). Partiendo de esta afirmación, la investigación en este campo se ha centrado tanto en las medidas para la prevención como en la posterior actuación. Por un lado, y como primer paso, estaría la prevención primaria, anteviendo dónde, cuándo y cómo puede ocurrir un accidente, y actuar para que no se produzca (Vignolo, Vacarezza, Álvarez, y Sosa, 2011). Por otro lado, y una vez acaecido éste, estaría la prevención secundaria, es decir, una reacción adecuada para paliar las consecuencias y minimizar la repercusión de los daños. Se sabe que, una práctica adecuada en primeros auxilios, entendido estos como los cuidados inmediatos y adecuados prestados a la persona accidentada antes de ser atendidos por auxiliares médicos, resulta imprescindible. Los primeros segundos nada más ocurrir un accidente, son fundamentales para el pronóstico posterior de las lesiones. Tal y como se recoge en la Guía para la Resucitación del Consejo Europeo de Resucitación (Monsieurs et al., 2015), una actuación correcta, in situ e inmediata, podría tanto atenuar las posteriores secuelas como salvar una vida. Según los datos de la Cruz Roja Chilena, gracias a la existencia de personas capacitadas en PA, un $25 \%$ de personas se salva de la muerte y un $38 \%$ no queda con serias secuelas (Chafloque, Pino, Rivera y Díaz, 2012).

Con todo, se puede decir que los accidentes escolares no parecen ser ni hechos aislados ni puntuales. Partiendo de la elevada tasa de ocurrencia de este tipo de accidentes, Miller y Shunk (1972) recalcan que la escuela tiene tres responsabilidades en lo referente a las emergencias y accidentes: 1) prevenir su ocurrencia; 2) instruir dentro del plan de estudios en materia de seguridad; y 3) ejecutar un programa de acción durante las emergencias en el contexto escolar.

Dentro del ámbito de la prevención primaria, son muchas las investigaciones que señalan que un número considerable de accidentes que ocurren en las escuelas se puede reducir. El conocimiento de las circunstancias que rodean a estos accidentes y sus factores de riesgo permitirían establecer estrategias preventivas adecuadas (Bruce y McGrath, 2005; Onís, Varona, Gil, Felici y Embid 2015; Towner y Scott 2008). Así, el estudio de Bena, Farina, Orengia y Quarta (2016), después de observar y analizar los accidentes acaecidos a lo largo de 5 años en escuelas italianas, evalúa la efectividad de los programas de seguridad implantados en las mismas, y así determina pautas de comportamientos y acciones seguras. De esta forma, desde el conocimiento de las circunstancias y los factores de riesgo, se establecen estrategias preventivas adecuadas (Longás et al., 2014). Por ejemplo: se diseñan guías para la prevención de accidentes en centros escolares (Estrada, Esteban, García y Llorente, 2015); se elaboran protocolos de actuación (Pennington, 2010); y se desarrollan directrices en PA (Van de Velde et al., 2007). Es decir, es necesario no sólo cuantificar los accidentes, sino también definirlos: conocer datos estadísticos de lugar de ocurrencia, de edad, de género o de tipo de siniestro. Al consultar tanto, las bases de datos del Instituto Nacional de Estadística (2014) como de Eustat (2013) no se ha encontrado información al respecto. Desafortunadamente, "la mayoría de clasificaciones no tienen en cuenta los accidentes escolares como una entrada específica" (Longás et al., 2014, p. 245). Gracias a los estudios realizados en el estado español sobre accidentes escolares se conocen algunas características de los mismos: aumentan con la edad de los escolares; son más frecuentes en chicos que en chicas; el lugar con mayor riesgo de accidentes es el patio; y las actividades que mayor riesgo conllevan son, las que se desarrollan en el recreo o durante la sesión de educación física (Brugulat, et al., 2009; Gautier y Martínez, 2009; Longás, et al., 2014; Osakidetza, 2013).

En cuanto a la prevención secundaria, una vez ocurrido el accidente, en la mayoría de los casos la persona que primero va a intervenir es la que esté presente en dicho instante. A nadie se le escapa que en el contexto educativo dos son las figuras centrales presentes en los diferentes momentos y lugares: el alumnado y el profesorado. Por lo general, cuando ocurre algún percance no hay ningún profesional sanitario en el centro 
(Navarrete, 2010) y los servicios de emergencia tardan una media de 5 minutos o más en llegar al lugar del accidente (Fothergill et al., 2013; Perkins et al., 2015). Sabiendo que el tiempo de respuesta es un factor decisivo y que el proporcionar una asistencia básica adecuada es crucial para evitar mayores complicaciones y salvar vidas (Lewis, Stubbs y Eisenberg, 2013), resulta fundamental que tanto el alumnado como el profesorado sean instruidos y estén capacitados en PA (Ammirati, Gagnayre, Amsallem, Némitz y Gignon 2014; Lockey y Georgiou, 2014).

En este sentido, son muchos los estudios que abordan la idea de la necesidad de que el alumnado esté formado y pueda actuar ante cualquier emergencia, de ahí que consideran, que la formación en PA debe ser asignatura obligada en todas las escuelas (Ammirati et al., 2014; Campbell, 2012). Reveruzzi, Buckley y Sheehan (2016) tras analizar 20 programas para la formación en PA a escolares, concluyen que la revisión de los mismos apoya la incorporación de los PA en el plan de estudios de la escuela. En la misma línea, tras el análisis de 30 estudios sobre educación en PA en escolares y adolescentes, De Buck et al. (2015) evidencian la necesaria integración de la formación en PA en la escuela. En el 2012, tan solo un $19 \%$ de los países europeos, incluyen dicha formación en el currículo de educación primaria o secundaria (Campbell, 2012). Investigaciones recientes sobre la instrucción al alumnado, señalan la falta de tiempo, la carencia de fondos, la presión curricular y/o la inexistencia de materiales de capacitación de maestros como barreras para implementar la formación por el profesorado en las escuelas (Hart, Flores, Brooks, Buick y Morrison, 2013; Lockey, Barton y Yoxall, 2015). De ahí, que ante la falta de formación (para formar), el profesorado prefiera que sean instructores externos los encargados de dar la formación al alumnado (Zinckernagel et al., 2016). En el estado español, la formación en PA se recoge como obligatoria con la Ley Orgánica 8/2013, de 9 de diciembre, para la mejora de la calidad educativa (LOMCE) y se hace explícita, con el Real Decreto 126/2014, de 28 de febrero, que establece el currículo básico de la Educación Primaria Obligatoria.

Por otra parte, todos los estudios centrados en la figura del profesorado apuntan que, es necesario que todo el profesorado esté formado en PA (Sönmez, Uskun y Pehlivan, 2014). Pese a que la necesidad está extendida a lo largo y ancho del planeta, los resultados obtenidos al avaluar el conocimiento y la capacitación del personal docente en este área no resultan nada alentadores: el conocimiento entre el profesorado en PA es bajo en cualquier lugar del mundo, tal y como apuntan los estudios llevados a cabo en Irlanda (Abernethy, MacAuley, McNally y McCann, 2003), en Italia (Bena, et al., 2016); en Shanghai (Li, Jiang, Jin, Qiu y Shen, 2012), en Turquía (Sönmez et al., 2014), en Polonia (Sosada, Zurawinski, Stepien, Makarska y Myrcik, 2002), en Iraq (Al-Robaiaay, 2013); en Arabia Saudi (Al-Samghan, Al-Shahrani y Al-Shahrani, 2015), en India, (Joseph, et al., 2015; Kumar, et al., 2013), en Estados Unidos (Gagliardi, Neighbors, Spears, Byrd y Snarr, 1994), en Grecia (Patsaki, et al., 2012); en Etiopía (Ganfure, 2016); y en Egipto (Mersal y Aly, 2016).

En el estado español, tan sólo un $12.5 \%$ de las universidades que ofertan el grado de Maestro o Maestra tiene una materia relacionada con los PA (Navarro, Penelas y Basanta, 2016). En el trabajo de Jiménez, Ramos y Ávila (2011) tras analizar las memorias de verificación de los títulos de maestro de primaria de 12 universidades españolas, se concluye que dos tercios se adaptan íntegramente a los objetivos y competencias que regula la Orden ECl/3857/2007, de 27 de diciembre. Atendiendo a las diferentes competencias que se mencionan en el estudio, ninguna de las doce universidades incluye alguna competencia relacionada con la formación en PA. Siendo la Universidad del País Vasco una de las universidades analizadas, cabe esperar que el profesorado que en la actualidad esta en activo en los centros escolares desconozca los conocimientos mínimos necesarios para una atención directa del accidentado y, no esté cualificado para, tal y como indica el Real Decreto 126/2014, instruir dicha materia a su alumnado. Con ello, este estudio tiene un triple objetivo: (a) analizar el grado de conocimiento que en PA posee el profesorado en activo de educación infantil y de primaria; (b) identificar aquellas variables que puedan influir en dicho conocimiento: género, edad, antigüedad, etapa educativa, especialidad, titulación académica y formación en PA; y (c) determinar una formación eficaz del profesorado a partir del análisis de diferentes perfiles del profesorado atendiendo a su conocimiento en PA.

\section{MATERIALES Y MÉTODOS}

Este trabajo se enmarca en un estudio diagnóstico de carácter descriptivo que tiene como objeto evaluar el conocimiento del profesorado de infantil y primaria sobre PA. Para ello se aplica una adaptación del cuestionario-test ad hoc semi-estandarizado previamente validado y utilizado por Abraldes y Ortín (2008), que queda compuesto por dos bloques. En el primero, se presentan preguntas abiertas y cerradas que hacen referencia a las variables independientes: genero, edad, ciclo, estudios, años de experiencia y formación en PA. Y en el segundo, se presenta una serie de preguntas cerradas sobre conocimientos en PA agrupadas en cuatro campos: a) Reanimación Cardio-Pulmonar (RCP), 7 preguntas; b) Traumatismos y contusiones (Trauma), 7 preguntas; c) Heridas y hemorragias (Heridas), 7 preguntas; y d) Otros (insolaciones, hipoglucemias, vómitos, asfixia, trauma dental, convulsiones), 6 preguntas. Se otorga "1" punto a las 
respuestas correctas y "0" puntos a las incorrectas. El total de respuestas correctas suma un máximo de 27 puntos. Tras calcular las puntuaciones totales de cada participante se categoriza su conocimiento en PA atendiendo a cuatro rangos: 0-9 puntos, muy deficientes; 10-13 puntos, deficiente; 14-24 puntos, aprobado; y 25-27 puntos, excelente. Para determinar el conocimiento en cada uno de los campos analizados se establecen los siguientes rangos para los campos relativos a RCP, Trauma y Heridas: 0-1 punto, muy deficiente; $2-3$ puntos deficiente; 4-5-6 puntos, aprobado: y 7 puntos excelente. El campo correspondiente a "Otros", al constar de seis preguntas, queda categorizado de la siguiente manera: 0 puntos, muy deficiente; 1-2 puntos, deficiente; 3-4-5 puntos, aprobado; y 6 puntos, excelente. Para la realización de este estudio, se añade una última batería de preguntas abiertas entre las que cabe destacar si durante sus años como docentes han tenido alguna experiencia en la que haya sido necesaria una actuación en PA y la percepción de la importancia que tienen los PA en relación a su trabajo.

La muestra está compuesta por 361 docentes de 17 centros públicos de educación infantil y primaria de la provincia de Bizkaia (norte de España), lo que supone una muestra representativa de dicho colectivo para un nivel de confianza del $95 \%$ y un margen de error de \pm 5.02 . La diferencia entre hombres $(15,2 \%)$ y mujeres $(84,8 \%)$ de la muestra se explica por el porcentaje de maestros $(13,79 \%)$ y maestras $(86,31 \%)$ existentes en el total de la población investigada en el curso 2013-2014 (Eustat, 2013). Las características de las y los participantes se recogen en la Tabla 1.

Tabla 1: Características descriptivas del profesorado participante $(n=361)$

\begin{tabular}{|l|l|c|c|}
\hline \multicolumn{1}{|c|}{ Rasgos } & \multicolumn{1}{|c|}{ Categorías } & $n$ & $\%$ \\
\hline Género & Mujer & 306 & 84.8 \\
& Hombre & 55 & 15.2 \\
\hline Edad & $\leq 33(24-33)$ & 41 & 11.4 \\
& $34-53$ & 245 & 67.86 \\
& $\geq 54$ (54-64) & 75 & 20.8 \\
\hline Ciclo & Educación Infantil & 127 & 35.2 \\
& Educación Primaria & 176 & 48.7 \\
& Ambos ciclos & 43 & 11.9 \\
& Dirección & 15 & 4.15 \\
\hline Titulación & Diplomatura & 346 & 94.45 \\
& Licenciatura & 82 & 22.7 \\
& Master & 29 & 8.03 \\
\hline Experiencia & $\leq 9(1-9)$ & 57 & 15.8 \\
& $10-29$ & 210 & 58.17 \\
& $\geq 30(30-44)$ & 94 & 25.8 \\
\hline
\end{tabular}

Los datos se analizan con el paquete estadístico SPSS (versión 23.0). Se generan estadísticos descriptivos y análisis de frecuencia y porcentaje de las respuestas obtenidas tanto en el total del cuestionario como en cada una de sus partes. Se implementan análisis de Chi-cuadrado para comparar las puntuaciones obtenidas atendiendo a las variables independientes anteriormente citadas. Así mismo, se clasifica a los sujetos mediante análisis de conglomerados jerárquicos. A partir de la creación de los clústeres (grupos) según los conocimientos en PA, se implementan nuevos análisis para determinar diferencias que permitan una mayor adecuación del tipo de formación a cada grupo. El nivel límite para determinar diferencias significas se establece para todos los casos en $p<0.05$.

\section{RESULTADOS}

Tras el análisis de datos se observa que el $68.42 \%$ del profesorado que ha participado en el test tiene un conocimiento deficiente en materia de PA, a los que se suma un $3.60 \%$ con conocimientos muy deficientes (ver Tabla 2). Hay que señalar igualmente que cero sujetos contesta correctamente a todas las preguntas del test y sólo 2 docentes alcanzan la puntuación de excelente. Así mismo, se constata que los conocimientos sobre la forma de actuar ante diferentes dolencias varían considerablemente. Así, mientras que el 73,95\% del profesorado sabe cómo actuar ante accidentes de trauma (53.73\% aprobado y $20.22 \%$ excelente), más del $50 \%$ del colectivo tiene considerables carencias sobre la forma correcta de actuar ante heridas, casos en los que sea preciso la realización de RCP y otros accidentes. 
Tabla 2: Conocimiento del profesorado sobre primeros sobre PA

\begin{tabular}{|l|c|c|c|c|c|c|c|c|}
\hline \multirow{2}{*}{ Puntuación } & \multicolumn{2}{|c|}{ M. Deficiente } & \multicolumn{2}{c|}{ Deficiente } & \multicolumn{2}{c|}{ Aprobado } & \multicolumn{2}{c|}{ Excelente } \\
\cline { 2 - 9 } & $N$ & $\%$ & $N$ & $\%$ & $N$ & $\%$ & $N$ & $\%$ \\
\hline Total & 13 & 3.60 & 247 & 68.42 & 99 & 27.42 & 2 & 0.55 \\
\hline RCP & 103 & 28.53 & 226 & 62.60 & 29 & 8.03 & 3 & 0.83 \\
\hline Trauma & 8 & 2.21 & 86 & 23.82 & 194 & 53.73 & 73 & 20.22 \\
\hline Heridas & 48 & 13.29 & 154 & 42.65 & 130 & 36.01 & 29 & 8.03 \\
\hline Otros & 20 & 5.54 & 197 & 54.57 & 137 & 37.95 & 7 & 1.93 \\
\hline
\end{tabular}

En cuanto al análisis con respecto a diversas variables independientes, se observa que el nivel de conocimiento no está asociado a la edad, el género, la etapa educativa, los años de experiencia como docente o la formación recibida (titulación). En cambio, sí existen diferencias significativas entre aquellas personas que han recibido formación en PA (166 participantes) y aquellas que no (ver Tabla 3). Aunque en ninguno de los dos grupos el $50 \%$ del profesorado llega a superar el test, en el grupo que ha recibido formación, hay más sujetos que superan la prueba (35.5\% aprobados y $0.6 \%$ excelente).

Tabla 3: Diferencias en la puntuación obtenida atendiendo a la formación en PA

\begin{tabular}{|c|c|c|c|c|c|c|c|c|c|c|}
\hline \multirow{3}{*}{$\begin{array}{c}\text { Formación } \\
\text { PA }\end{array}$} & \multicolumn{8}{|c|}{ Puntuación test } & & \\
\hline & \multicolumn{2}{|c|}{ M. deficiente } & \multicolumn{2}{|c|}{ Deficiente } & \multicolumn{2}{|c|}{ Aprobado } & \multicolumn{2}{|c|}{ Excelente } & \multicolumn{2}{|c|}{ Chi Cuadrado } \\
\hline & $\mathrm{N}$ & $\%$ & $\mathrm{~N}$ & $\%$ & $\mathrm{~N}$ & $\%$ & $\mathrm{~N}$ & $\%$ & Pearson & Sign. \\
\hline No & 9 & 4.6 & 145 & 74.4 & 40 & 20.5 & 1 & 0.5 & \multirow{2}{*}{10.795} & \multirow{2}{*}{.013} \\
\hline Sí & 4 & 2.4 & 102 & 61.4 & 59 & 35.5 & 1 & 0.6 & & \\
\hline Total & 13 & 3.6 & 247 & 68.4 & 99 & 27.4 & 2 & 0.6 & & \\
\hline
\end{tabular}

Entre aquellas personas que poseen formación, existen diferencias considerables en función del tiempo transcurrido desde que recibieron la formación. Así, hay más sujetos que superan el test entre aquellos que realizaron la formación en los últimos cinco años frente a quienes lo hicieron hace más de cinco años. Sin embargo estas diferencias no pueden ser consideradas estadísticamente significativas (Pearson: 7.32 y Sign: 0.62) dentro del límite fijado en este estudio.

Tras realizar un análisis de conglomerados jerárquicos se obtienen 4 clústeres. Estos clasifican a los sujetos en otros tantos grupos que permiten profundizar en los resultados. Así, se genera un primer grupo minoritario (grupo 1) que aglutina al $18.60 \%$ de la muestra, un segundo grupo mayoritario con un $79.80 \%$ (grupo 2 ) y dos grupos residuales, uno de 4 sujetos (grupo 3) y otro de 2 sujetos (grupo 4).

Tabla 4: Agrupamiento del profesorado según el conocimiento en PA

\begin{tabular}{|c|c|c|c|c|c|c|}
\hline Grupo & Estadístico & Muy Deficiente & Deficiente & Aprobado & Excelente & Total \\
\hline \multirow{3}{*}{1} & Recuento & 0 & 0 & 67 & 0 & 67 \\
\hline & $\%$ Grupo & 0 & 0 & 100 & 0 & 100 \\
\hline & $\%$ del total & 0 & 0 & 18.60 & 0 & 18.60 \\
\hline \multirow{3}{*}{2} & Recuento & 9 & 247 & 32 & 0 & 288 \\
\hline & $\%$ Grupo & 3.10 & 85.80 & 11.10 & 0 & 100 \\
\hline & $\%$ del total & 2.50 & 68.40 & 8.90 & 0 & 79.80 \\
\hline \multirow{3}{*}{3} & Recuento & 4 & 0 & 0 & 0 & 4 \\
\hline & $\%$ Grupo & 100 & 0 & 0 & 0 & 100 \\
\hline & $\%$ del total & 1.10 & 0 & 0 & 0 & 1.10 \\
\hline \multirow{3}{*}{4} & Recuento & 0 & 0 & 0 & 2 & 2 \\
\hline & \% Grupo & 0 & 0 & 0 & 100 & 100 \\
\hline & $\%$ del total & 0 & 0 & 0 & 0 & 0.60 \\
\hline \multirow{3}{*}{ Total } & Recuento & 13 & 247 & 99 & 2 & 361 \\
\hline & $\%$ Grupo & 3.60 & 68.40 & 27.40 & 0.60 & 100 \\
\hline & $\%$ del total & 3.60 & 68.40 & 27.40 & 0.60 & 100 \\
\hline
\end{tabular}


En la Tabla 4, se constata que los dos grupos residuales son opuestos en cuanto a los resultados obtenidos en el test. Mientras el grupo 3 está compuesto por cuatro sujetos que han obtenido una calificación muy deficiente en el test, los dos sujetos del grupo 4 han obtenido la calificación de excelente. Son por lo tanto, antagónicos. Desestimados los grupos 3 y 4 por el bajo número de sujetos que lo componen, se han buscado diferencias significativas entre los grupos 1 y 2. Tras analizar la distribución de los sujetos según su resultado, se observa que el $100 \%$ del profesorado del grupo 1 supera el test final mientras que sólo el $11 \%$ del grupo 2 (el más mayoritario) lo consigue.

Tabla 5: Diferencias en los resultados obtenidos según grupos de profesorado

\begin{tabular}{|c|c|c|c|c|c|c|c|c|c|c|c|}
\hline \multirow[t]{2}{*}{ Área } & \multirow[t]{2}{*}{ Grupo } & \multicolumn{2}{|c|}{ Muy deficiente } & \multicolumn{2}{|c|}{ Deficiente } & \multicolumn{2}{|c|}{ Aprobado } & \multicolumn{2}{|c|}{ Excelente } & \multicolumn{2}{|c|}{ Chi Cuadrado } \\
\hline & & $N$ & $\%$ & $N$ & $\%$ & $N$ & $\%$ & $N$ & $\%$ & Pearson & Sign. \\
\hline \multirow{2}{*}{$\mathrm{RCP}$} & Grupo1 & 6 & 9.0 & 46 & 68.7 & 13 & 19.4 & 2 & 3.0 & \multirow{2}{*}{33.421} & \multirow{2}{*}{.000} \\
\hline & Grupo2 & 93 & 32.3 & 108 & 62.5 & 15 & 5.2 & 0 & 0.6 & & \\
\hline \multirow{2}{*}{ Traumas } & Grupo1 & 0 & 0 & 2 & 3.0 & 36 & 53.7 & 29 & 43.3 & \multirow{2}{*}{38.712} & \multirow{2}{*}{.000} \\
\hline & Grupo2 & 4 & 1.4 & 84 & 29.2 & 158 & 54.9 & 42 & 14.6 & & \\
\hline \multirow{2}{*}{ Heridas } & Grupo1 & 0 & 0 & 11 & 16.4 & 33 & 49,3 & 23 & 34.3 & \multirow{2}{*}{101.072} & \multirow{2}{*}{.000} \\
\hline & Grupo2 & 44 & 15.3 & 143 & 49.7 & 96 & 33,3 & 5 & 1.7 & & \\
\hline \multirow{2}{*}{ Otros } & Grupo1 & 0 & 0 & 15 & 22.4 & 47 & 70.1 & 5 & 7.5 & \multirow{2}{*}{58.169} & \multirow{2}{*}{.000} \\
\hline & Grupo2 & 16 & 5 & 182 & 63.2 & 89 & 30.9 & 1 & 0.3 & & \\
\hline \multirow{2}{*}{ Total test } & Grupo1 & 0 & 0 & 0 & 0 & 67 & 100 & 0 & 0 & \multirow{2}{*}{213.588} & \multirow{2}{*}{.000} \\
\hline & Grupo2 & 9 & 3.1 & 247 & 85.8 & 32 & 11.1 & 0 & 0 & & \\
\hline
\end{tabular}

Pese a los buenos datos del grupo 1 (aquellos que consiguen superar el test), existen importantes carencias en cada una de las áreas de conocimiento evaluadas. Así, en este grupo, el 68,7\% tiene conocimientos deficientes (y un $9 \%$ muy deficientes) sobre la forma de actuar en la RCP. En cambio, estos porcentajes descienden y oscilan entre el $3.0 \%$ para el caso de traumas, $16.4 \%$ para heridas y el $22.4 \%$ para otro tipo de actuaciones, no presentando ningún sujeto de este grupo conocimientos muy deficientes para estas tres áreas. Por lo tanto hay que destacar que, incluso quienes mayor conocimientos de PA tienen (grupo 1), poseen graves carencias sobre RCP.

Un estudio más detallado muestra que no se han encontrado diferencias significativas en cuanto a las características de cada grupo; así su distribución en cuanto a género, titulación, ciclo y zona es similar, y las pequeñas variaciones pueden ser atribuidas al azar. En cambio, al igual que en los resultados generales, sí se observan diferencias significativas (Pearson: 9.034 y Sign.: 0.000) en la composición de los grupos 1 y 2 atendiendo a la realización previa de cursos de PA. El 62.7\% de los sujetos del grupo 1 -que logra mejores puntuaciones en el test- tienen realizado un curso previo sobre PA frente a un $42.4 \%$ del grupo dos.

Por último, y en lo que respecta a las preguntas de opinión que cierran el cuestionario, hay que destacar que el $86,7 \%$ de los 313 docentes que componen la muestra, está interesado en el tema de PA y que 305 (84.5\%) consideran de mucha importancia dicho conocimiento en relación a su trabajo. Aunque el profesorado de educación física se muestra más interesado que el resto y considera más importante tener un buen conocimiento en el tema, no se han podido establecer diferencias significativas (Pearson: 4.149 y Sign.: 0.126) entre profesores de diferentes especialidades dentro de los límites establecidos en el estudio. Así mismo, cabe constatar que un $23.8 \%$ del profesorado de educación infantil y primaria ha tenido alguna experiencia en la que un mínimo conocimiento en PA hubiera solucionado o agilizado alguna actuación acaecida en el centro escolar.

\section{DISCUSIÓN}

Como se ha dicho, los accidentes en el rango de edad escolar no son hechos aislados. Debido a que los niños y niñas pasan la mayor parte del día en la escuela, el profesorado debe ser competente en las habilidades básicas de PA. No obstante, el conocimiento en PA de los y las docentes de educación infantil y primaria participantes en este estudio es bajo. La falta de conocimiento se podría explicar por la carencia de una asignatura relacionada con los PA en las titulaciones universitarias que dan acceso al título de maestro. Los datos concuerdan con otras investigaciones realizadas en otros países en las que el profesorado tampoco demuestra conocimientos suficientes en PA. En el estudio de Al-Robaiaay (2013) tan sólo un 4\% del profesorado de primaria tiene buen conocimiento en PA frente al $77 \%$ que presenta un conocimiento deficitario.

Kumar et al. (2013) encuentran que existen diferencias significativas en relación a la titulación y a los años de experiencia, siendo el profesorado de educación física y aquellos que tienen más de 10 años de experiencia quienes puntúan significativamente mejor que el resto. Sin embargo, Al-Robaiaay (2013) no encuentra estas 
diferencias, hecho que atribuye a un descuido generalizado en la formación en PA de todo el profesorado. En el presente estudio, el hecho de que tampoco se observen diferencias significativas en estas variables, sumado a que tan solo un $27.97 \%$ de la muestra conteste correctamente más del $50 \%$ de las preguntas, nos puede llevar a pensar lo mismo. En cuanto a la edad de los y las docentes, los resultados del estudio difieren en los hallados en estudios similares. Así, en el estudio de Patsaki et al. (2012) se observa que según aumenta la edad, disminuye la probabilidad de una actuación correcta en PA.

En este estudio actual, pese a que un $46 \%$ de la muestra ha recibido previamente formación en PA, sólo un $27.42 \%$ tiene conocimientos adecuados en PA y tan sólo un $16.62 \%$ considera que tiene un nivel bueno sobre los mismos. Datos similares se encuentran en el estudio Sönmez et al. (2014), en el que un $73 \%$ del profesorado de infantil ha recibido algún curso de formación y tan sólo el $15.5 \%$ considera que su conocimiento es adecuado. Realidad que se podría explicar bien a través del control de la calidad y cantidad del contenido de los cursos recibidos, bien atendiendo al tiempo pasado desde la realización del mismo (Martín, 2015). Joseph et al. (2015) encuentran que, el tiempo pasado desde la última formación resultaba significativo. Todo ello hace pensar que, con el tiempo lo aprendido se olvida y que es necesaria un formación continua y permanente en esta materia. Necesidad también recogida por el Consejo Europeo de Resucitación de Reanimación (Ballance et al., 2015) al decir que, la formación en PA debe repetirse periódicamente en "pequeñas dosis" con el fin de mantener niveles adecuados de habilidad y experiencia. Tanto en este estudio como en el resto de estudios se observa que, los participantes que han recibido previamente cualquier tipo de formación en PA, puntúan mejor que los que no han recibido ninguna formación.

Además, de las 361 personas participantes en el estudio, 86 de ellas (23.8\%) en sus años como docente ha tenido alguna experiencia en la que un mínimo conocimiento en PA hubiera solucionado o agilizado alguna actuación acaecida en el centro escolar. Datos similares se recogen en otros estudios: un $65.1 \%$ del profesorado de educación infantil ha proporcionado PA en la escuela al menos una vez en su recorrido profesional (Slabe y Fink, 2013); un 50.8\% de los docentes de primaria (Al-Samghan et al., 2015); un 18\% del profesorado de primaria y secundaria (Sundblad, Saartok, Engström y Renström, 2005); y un 17\% de los docentes de las escuelas públicas de Arkansas, Kansas y Missouri, ha vivido al menos una emergencia que amenazaba con la vida de algún estudiante (Gagliardi et al., 1994). En el estudio de Joseph et al. (2015) se constata que un $80 \%$ del profesorado participante había administrado PA en situaciones reales, hecho que atribuyen a la buena predisposición y actitud positiva del profesorado para asistir en PA en caso de emergencia pese a no haber sido formados en la materia. Los datos también confirman que los accidentes escolares no parecen ser hechos aislados ni puntuales.

Finalmente, cabe destacar, que los y las participantes en este estudio son conscientes de la importancia de este conocimiento en el desarrollo de su labor como docente. A un $86.7 \%$ le interesa la formación en PA. Interés que también se recoge en el estudio de Martín (2015) al observar que un $78.9 \%$ de los asistentes a las sesiones educativas en PA lo hicieron de manera voluntaria.

\section{CONCLUSIONES}

La principal conclusión a la que llega este estudio es que los conocimientos que presenta el profesorado de infantil y primaria en PA son deficitarios y no garantizan dar una respuesta adecuada; así por ejemplo, tienen conceptos erróneos sobre cómo curar heridas y, sobre todo, desconocen la actuación correcta en RCP. Los conocimientos son mejores cuando se ha realizado algún curso de formación en PA. Con ello, este estudio muestra una necesidad urgente de formación en PA del profesorado en activo. Conclusión que se enmarca dentro de la prevención secundaria. Es necesario formar al profesorado para que éste tenga una reacción adecuada y así paliar las consecuencias y minimizar la repercusión de los daños. Además, la formación les va a proporcionar también aumento de la autoeficacia y de la voluntad de actuar ante un accidente (Oliver, Cooper y McKinney, 2014).

A partir de los resultados encontrados de los análisis de conglomerados jerárquicos realizados en el estudio, se recomienda establecer dos tipos de formación dirigidas a dos perfiles diferenciados de profesorado:

i) Una formación básica para todos aquellos docentes o estudiantes del grado de infantil y/o primaria. Fundamentalmente, estaría centrada en adquirir los conocimientos básicos sobre la actuación y la prevención de PA teniendo en cuenta los accidentes más frecuentes en los centros escolares (Estrada et al., 2015). Esta formación, se podría implementar de dos maneras: (1) tal y como señalan González y Reche (2010) mediante una formación complementaria al grado de maestro/a, dirigida a la adquisición de las competencias profesionales, especialmente para aquellos que no han realizado cursos de PA; o (2) dentro de los propios estudios de grado de maestro/a. En esta línea, el estudio de Cubero, Calderón, Costillo y Ruiz (2010), recomienda la inclusión de un itinerario en Prevención de accidentes y primeros auxilios en los estudios de 
grado, y aboga por su inclusión en los programas oficiales de postgrado. El estudio de Martín (2015) considera primordial que esta formación básica dirigida, tanto a docentes en activos como a estudiantes de grado, sea impartida por personal sanitario cualificado.

ii) Una formación continua dirigida al profesorado en activo. Tal y como recomienda el Consejo Europeo de Resucitación de Reanimación (Ballance et al., 2015) el objetivo es repasar y renovar periódicamente conocimientos previamente adquiridos sobre los modos de actuación ante traumas, heridas y otro tipo de lesiones, que a su vez se centre especialmente en RCP, ya que éste es el aspecto sobre el que más carencias se han encontrado entre aquellos que en su día realizaron una formación en PA.

Atendiendo al interés mostrado por la gran mayoría del profesorado, cabe esperar altos índices de participación en ambos tipos de formación, en la básica y en la continua. Ambos tipos de formación, capacitarían al profesorado para actuar ante cualquier accidente en el centro escolar. Pero además, y dado que, el Real Decreto 126/2014 obliga a las escuelas a introducir contenidos sobre PA, se considera necesaria un tercer tipo de formación: una formación para formar. Es decir, una formación que capacite al profesorado para trasmitir a su alumnado los conocimientos sobre PA que estos necesitan. Además, la entrada en vigor de nuevas leyes educativas, como la LOMCE en España, exigirá nuevas investigaciones en este campo. Unas, para determinar quién debe instruir dicha materia en la escuela, bien el propio docente bien un profesional sanitario; y otras, para determinar y posibilitar su desarrollo dentro del marco escolar: elaboración de materiales, diseño de programaciones didácticas, recursos, tiempo, fondos, etc.

El presente estudio, no sólo constata la necesidad de formación del profesorado sino que evidencia, además, la urgencia de elaborar medidas dentro del marco de la prevención primaria. Tal y como demuestran diferentes estudios (Estrada et al., 2015; Vignolo et al., 2011), es necesario conocer dónde, cuándo y cómo las probabilidades de ocurrir un accidente son mayores y actuar así, para que no se produzca. Por ello, se considera oportuno que tanto el Instituto Nacional de Estadística como el Instituto Vasco de Estadística (Eustat) incluyan esta información en sus bases de datos. Datos que, sin ninguna duda, servirán de herramienta para desarrollar e implementar programas para la prevención de accidentes en el ámbito escolar.

Para finalizar es conveniente recordar que el Committee on School Health (2001) señala que el profesorado debe estar formado en PA, pues es éste quien debe actuar ante un accidente en el centro escolar. Para ello, es necesario que el profesorado reciba una formación inicial básica en PA, y renueve sus conocimientos y habilidades en cursos de formación continuada, logrando así una actuación correcta y eficaz ante cualquier tipo de percance. Un profesorado bien capacitado en PA puede, además, formar a su alumnado en esta materia.

\section{REFERENCIAS}

Abernethy, L., MacAuley, D., McNally, O. y McCann, S., Immediate Care of School Sport Injury, doi: 10.1136/ip.9.3.270, Injury Prevention, 9, 270-273 (2003)

Abraldes, J. A. y Ortín, A., La Formación del Profesorado de Educación Física en Primeros Auxilios. APEF_ Almería, España (2008)

Al-Robaiaay, Y. K., Knowledge of Primary School Teachers Regarding First Aid in Baghdad, Al-Kindy College Medical Journal, 9, 54-59 (2013)

Al-Samghan, A. S., Al-Shahrani, F. M. y Al-shahrani, F. H., Primary School Teachers' Knowledge about FirstAid, http://medicaljournalofcairouniversity.net/home2/images/pdf/2015/June/68.pdf, Medical Journal of Cairo University, 83(1), 541-547 (2015)

Ammirati, C., Gagnayre, R., Amsallem, C., Némitz, B., y Gignon, M., Are Schoolteachers Able to Teach First Aid to Children Younger than 6 Years? A comparative study, https://goo.gl/Qkxqgp, BMJ open, 4(9), e005848 (2014)

Ballance, J. H., Barelli, A., Biarent, D., Bossaert, L., Castrén, M., Handley, A. J., y Raffay, V., European Resuscitation Council Guidelines for Resuscitation, Resuscitation, (95), 288-301 (2015)

Bena, A., Farina, E., Orengia, M., y Quarta, D., Promotion of Safety Culture in Italian Schools: Effectiveness of Interventions on Student Injuries, doi: 10.1093/eurpub/ckw039, The European Journal of Public Health, ckw039 (2016)

Bruce, B. y McGrath, P., Group Interventions for the Prevention of Injuries in Young Children: A Systematic Review, doi:10.1136/ip.2004.007971, Injury Prevention, 11, 143-147 (2005) 
Brugulat, P., Medina, A. y Mompart, A. (Eds.). La Salut de la Població Infantil a Catalunya: Enquesta de Salut de Catalunya 2006, Barcelona, Generalitat de Catalunya, Departament de Salut (2009)

Campbell, S., Supporting Mandatory First Aid Training in Primary Schools, Nursing Standar, 27(6), 35-9 (2012)

Chafloque, J., Pino, M., Rivera, M. y Díaz, C., Conocimientos Adecuados de Emergencias Médicas: Un Problema de Estudiantes y Profesionales de la Salud, http://scielo.isciii.es/pdf/edu/v15n1/carta2.pdf, Educación Médica, 15(1), 11-12 (2012)

Committee on School Health, Guidelines for Emergency Medical Care in School, Pediatrics, 107, 435-436 (2001)

Comunidad Valenciana, Encuesta de Salud de la Comunitat Valenciana, Generalitat Valenciana, https://goo.gl/CWmVd4, ISBN: 978-84-482-5746-0 (2010)

Cubero, J., Calderón, M., Costillo, E. y Ruiz, C., La Educación para la Salud en el Espacio Europeo de Educación Superior, Universidad de Granada. https://goo.gl/nl100D, Publicaciones, 41, 51-63 (2011)

De Buck, E., Van Remoortel, H., Dieltjens, T., Verstraeten, H., Clarysse, M., Moens, O. y Vandekerckhove, P., Evidence-based Educational Pathway for the Integration of First Aid Training in School Curricula, doi: 10.1016/j.resuscitation.2015.06.008, Resuscitation, 94, 8-22 (2015)

Estrada, C., Esteban, C., García, P., y Llorente, B., Guía para la Prevención de Centros Escolares, http://faros.hsjdbcn.org/adjuntos/348.1-guia_prevencion_accidentes_escolares.pdf, Madrid (2015)

Eustat, Eskola-jardueraren estatistika 2013/2014 ikasturtea, https://goo.gl/j7vUgP, (2013)

Fothergill, R. T., Watson, L. R., Chamberlain, D., Virdi, G. K., Moore, F. P., y Whitbread, M., Increases in Survival from Out-Of-Hospital Cardiac Arrest: A Five Year Study, doi: 10.1016/j.resuscitation.2013.03.034, Resuscitation, 84(8), 1089-1092 (2013)

Gagliardi, M., Neighbors, M., Spears, C., Byrd, S. y Snarr, J., Emergencies In the School Setting: Are Public School Teachers Adequately Trained to Respond? Prehospital and Disaster Medicine, 9, 222-225 (1994)

Ganfure, G., First Aid Knowledge, Attitude and Practice among Kindergarten Teachers of Lideta-Sub-City, Addis Ababa, Ethiopia, http://etd.aau.edu.et/bitstream/123456789/10192/1/Gemechu\%20Ganfure.pdf Doctoral dissertation, AAU (2016)

Gautier, M. C., y Martínez, V., Accidentes y Lesiones más Frecuentes en Edad Escolar en Colegios de Santander y Cantabria, https://goo.gl/fKDMj3, Revista Nuberos (2009)

González, I., Reche, E., Las Demandas Formativas del Alumnado de Magisterio. Construcción de un Plan de Formación Complementaria, http://www.javeriana.edu.co/magis, Magis: Revista Internacional de Investigación en Educación, 2(4), 383-400 (2010)

Hart, D., Flores, O., Brooks, S., Buick, J. E., y Morrison, L. J., Cardiopulmonary Resuscitation and Automatic External Defibrillator Training in Schools: Is Anyone Learning How To Save A Life?, Cjem, 15(05), 270-278 (2013)

Instituto Nacional de Estadística, Encuesta Nacional de Salud, http://www.ine.es/, Madrid, Autor (2014)

Jiménez, L., Ramos, F. J., y Ávila, M., Las Universidades Españolas y EEES: Un Estudio sobre los Títulos de Grado de Maestro en Educación Primaria, doi: 10.4067/S0718-50062012000100005, Formación Universitaria, 5(1), 33-44 (2012)

Joseph, N., Narayanan, T., bin Zakaria, S., Venugopal Nair, A., Belayutham, L. y Subrmanian, A. M., Awareness, Attitudes and Practices of First Aid Among School Teachers in Mangalore, South India, Journal of Primary Health Care, 7(4), 274-281 (2015)

Kumar, S. D., Kulkarni, P., Srinivas, N., Prakash, B., Hugara, S. y Ashok, N.C., Perception and Practices Regarding First-Aid Among School Teachers in Mysore, National Journal of Community Medicine, 4, 349-52 (2013)

Lewis, M., Stubbs, B. A., y Eisenberg, M. S., Dispatcher-assisted CPR: Time to Identify Cardiac Arrest and Deliver Chest Compression Instructions, doi: 10.1161/CIRCULATIONAHA.113.002627, Circulation, 113 (2013)

Ley Orgánica 8/2013, de 9 de diciembre, para la Mejora de la Calidad Educativa (LOMCE). Boletín Oficial del Estado, http://www. boe. es/diario_boe/txt. php, acceso 7 de febrero de 2016 (2013) 
Li, F., Jiang, F., Jin, X., Qiu, Y. y Shen, X., Pediatric First Aid Knowledge and Attitudes Among Staff in the Preschools of Shanghai, China, doi: 10.1186/1471-2431-12-121, BMC Pediatrics, 12(121), 1-7 (2012)

Lockey A.S., Barton, K. y Yoxall, H., Opportunities and Barriers to Cardiopulmonary Resuscitation Training in English Secondary Schools, doi: 10.1097/MEJ. 0000000000000307, Eur. J. Emerg. Med. (2015)

Lockey, A.S., y Georgiou, M., Children Can Save Lives, doi: 10.1016/j.resuscitation.2013.01.011, Resuscitation, 84(4), 399-400 (2013)

Longás, E., Longás, J. y Riera i Romaní, J., Daños No Intencionados en la Escuela. Estudio de su Incidencia en Escolares de Cataluña, doi: http://dx.doi.org/10.5565/rev/educar.42, Educar, 50(2), 243-263 (2014)

Mapfre, Accidentes de la Población Infantil Española. Área de Salud y Prevención, https://goo.gl/GQ5AF3, Asociación española de Pediatría de Atención Primaria (2014)

Martín, R. A., Educación para la Salud en Primeros Auxilios Dirigida al Personal Docente del Ámbito Escolar, doi: 10.1016/j.reu.2015.04.004, Enfermería Universitaria, 12(2), 88-92 (2015)

Mersal, F. A., y Aly, R. A. E. S., Developing Disaster Management and First Aid Guidelines for School Teachers in Cairo Egypt, doi: 10.5430/jnep.v6n7p41, Journal of Nursing Education and Practice, 6(7), 41 (2016)

Miller, D. F. y Shunk, S., A Survery of Elementary School Health Services with Emphasis on Preparation for Emergency Care Procedures of Sick And Injured Students, The Journal of School Health, 42(2), 114-117 (1972)

Monsieurs, K. G., Zideman, D. A., Alfonzo, A., Arntz, H. R., Askitopoulou, H., Bellou, A., y Böttiger, B. W., European Resuscitation Council Guidelines for Resuscitation 2015: section 1. Executive summary. https://goo.gl/cFGm6D (2015)

Navarrete, R., La Actuación del Docente en Educación Física ante el Accidente Escolar, https://goo.gl/J0Lj3g, Revista Portaldeportivo, 4,1-15 (2010)

Navarro, R., Penelas, D. y Basanta, S., ¿Tienen las Futuras Maestras y Maestros de Educación Primaria la Formación Necesaria para Iniciar las Maniobras de Reanimación Cardiopulmonar en Caso de Emergencia Escolar? Un estudio descriptivo, doi: 10.5565/rev/educar.714, Educar, 52(1) 149-168 (2016)

Oliver, E., Cooper, J., y McKinney, D., Can First Aid Training Encourage Individuals' Propensity to Act in An Emergency Situation? A Pilot Study, doi: 10.1136/emermed-2012-202191 Emergency Medicine Journal, 31, 518-120 (2014)

Onís, E., Varona, I., Gil, M., Felici, C., y Embid, P., Lesiones No Intencionadas en el Centro Escolar: ¿De qué Estamos Hablando?, https://goo.gl/8jvRQA, Pediatría Atención Primaria, 17(68), 333-339 (2015)

Orden ECI/3857/2007, de 27 de diciembre, por la que se Establecen los Requisitos para la Verificación de Los Títulos Universitarios Oficiales que Habiliten para el Ejercicio de la Profesión de Maestro en Educación Primaria, BOE 312/2007 de 29 de diciembre (2007)

Osakidetza, Encuesta de Salud del País Vasco, https://goo.gl/lenbPJ, Gobierno Vasco (2013)

Patsaki, A., Pantazopoulos, I., Dontas, I., Passali, C., Papadimitriou, L. y Xanthos, T., Evaluation of Greek High School Teachers' Knowledge in Basic Life Support, Automated External Defibrillation, and Foreign Body Airway Obstruction: Implications For Nursing Interventions, Journal of Emergency Nursing, 38(2), 176-181 (2012)

Pennington, N., Head Injuries in Children, Journal of School Nursing, 26(1), 26-32 (2010)

Perkins, G. D., Lall, R., Quinn, T., Deakin, C. D., Cooke, M. W., Horton, J., y Smyth, M., Mechanical Versus Manual Chest Compression for Out-Of-Hospital Cardiac Arrest (PARAMEDIC): A Pragmatic, Cluster Randomised Controlled Trial, doi: 10.1016/S0140-6736(14)61886-9, The Lancet, 385(9972), 947-955 (2015)

Real Decreto 126/2014, de 28 de febrero, por el que se establece el Currículo Básico de la Educación Primaria. Boletín Oficial del Estado, 52, 19349-19420 (2014)

Reveruzzi, B., Buckley, L. y Sheehan, M., School-Based First Aid Training Programs: A Systematic Review, Journal of School Health, 86(4), 266-272 (2016) 
Slabe, D. y Fink, R., Kindergarten Teachers' and Their Assistants' Knowledge of First Aid in Slovenian Kindergartens, Health Education Journal, 72(4), 398-407 (2013)

Sönmez, Y., Uskun, E. y Pehlivan, A., Knowledge Levels of Pre-School Teachers Related with Basic First-Aid Practices, Isparta Sample, doi: 10.5152/tpa.2014.1581, Turkish Archives of Pediatrics, 49, 238-246 (2014)

Sosada, K., Zurawiński, W., Stepień, T., Makarska, J. y Myrcik, D., Evaluation of the Knowledge Of Teachers and High School Students in Silesia on the Principles of First Aid, Wiadomosci lekarskie (Warsaw, Poland: 1960), 55(2), 883-889 (2001)

Sundblad, G., Saartok, T., Engström, L. M. y Renström, P., Injuries During Physical Activity in School Children, Scandinavian Journal of Medicine and Science in Sports, 15(5), 313-323 (2005)

Towner, E. y Scott, I., World Report on Child on Injury Preventios: Chapter 1, Child Injuries in Context, http://eprints.qut.edu.au/17140/2/9789241563574_eng.pdf, ISBN 978924156357 4, Switzerland, World Health Organization (2008)

Van de Velde, S., Broos, P., Van Bouwelen, M., De Win, R., Sermon, A., Verduyckt, J., y Van den Steene, P. European First Aid Guidelines, doi: 10.1016/j.resuscitation.2006.10.023, Resuscitation, 72(2), 240-251 (2007)

Vignolo, J., Vacarezza, M., Álvarez, C., y Sosa, A., Niveles de Atención, de Prevención y Atención Primaria de la Salud, http://www.scielo.edu.uy/pdf/ami/v33n1/v33n1a03.pdf, ISSN 0250-3816, Archivos de Medicina Interna, 33(1), 11-14 (2011)

World Health Organization, Child and Adolescent Injury Prevention: A WHO Plan of Action 2006-2015, Department of Injuries and Violence Prevention, https://goo.gl/uWYskZ, Switzerland (2006)

Zinckernagel, L., Hansen, C. M., Rod, M. H., Folke, F., Torp-Pedersen, C., y Tjørnhøj-Thomsen, T., What Are the Barriers to Implementation of Cardiopulmonary Resuscitation Training in Secondary Schools? A Qualitative Study, doi:10.1136/bmjopen-2015- 010481, BMJ open, 6(4), e010481 (2016) 
